\title{
Turbulence, feedback, and slow star formation
}

\author{
Mark R. Krumholz ${ }^{1}$ \\ ${ }^{1}$ Department of Astrophysical Science, Princeton University, Peyton Hall, Ivy Lane, Princeton, \\ NJ 08544, USA \\ email: krumholz@astro.princeton.edu
}

\begin{abstract}
One of the outstanding puzzles about star formation is why it proceeds so slowly. Giant molecular clouds convert only a few percent of their gas into stars per free-fall time, and recent observations show that this low star formation rate is essentially constant over a range of scales from individual cluster-forming molecular clumps in the Milky Way to entire starburst galaxies. This striking result is perhaps the most basic fact that any theory of star formation must explain. I argue that a model in which star formation occurs in virialized structures at a rate regulated by supersonic turbulence can explain this observation. The turbulence in turn is driven by star formation feedback, which injects energy to offset radiation from isothermal shocks and keeps star-forming structures from wandering too far from virial balance. This model is able to reproduce observational results covering a wide range of scales, from the formation times of young clusters to the extragalactic IR-HCN correlation, and makes additional quantitative predictions that will be testable in the next few years.
\end{abstract}

Keywords. turbulence, stars: formation, ISM: clouds, galaxies: ISM, galaxies: starburst

\section{Introduction}

Zuckerman \& Evans (1974) were the first to point out perhaps the most surprising fact about star formation: it is remarkably slow. Inside the solar circle there are roughly $M_{\text {mol }} \approx 10^{9} M_{\odot}$ of molecular gas (Bronfman et al. 2000), organized into giant molecular clouds (GMCs) with typical densities of $\sim 100 \mathrm{H}$ atoms $\mathrm{cm}^{-3}$, giving a free-fall time of about $t_{\mathrm{ff}} \approx 4 \mathrm{Myr}$ (McKee 1999). However, the star formation rate in the Milky Way is only $\sim 3 M_{\odot} \mathrm{yr}^{-1}$ (McKee \& Williams 1997), vastly less than the rate of $\sim 250 M_{\odot}$ $\mathrm{yr}^{-1}$ that one would expect if molecular clouds were converting their mass into stars on a free-fall time scale. More recent observations of nearby Milky Way-like galaxies (Wong \& Blitz 2002) find that this factor of $~ 100$ discrepancy occurs in them too. Nor is the discrepancy any smaller in systems like ULIRGs with much larger star formation rates. For example, Downes \& Solomon (1998) find that Arp 220 contains roughly $2 \times 10^{9} M_{\odot}$ of molecular gas with a typical free-fall time of $\sim 0.5 \mathrm{Myr}$, but the observed star formation rate of $\sim 50 M_{\odot} \mathrm{yr}^{-1}$ is a factor of 100 smaller than $M_{\mathrm{mol}} / t_{\mathrm{ff}}$.

Recently, Krumholz \& Tan (2006) pointed out that objects much denser than GMCs form stars just as slowly. If one repeats the Zuckerman \& Evans (1974) calculation of dividing total mass by characteristic free-fall time for any class of dense, gaseous objects (e.g. infrared dark clouds, dense molecular clumps), one again obtains a rate roughly 100 times larger than the observed star formation rate. This is true in galaxies from normal spirals to ULIRGs, and for objects with densities from $\sim 100 \mathrm{~cm}^{-3}$, typical of GMCs, to $\sim 10^{4}-10^{5} \mathrm{~cm}^{-3}$, typical of molecular clumps forming rich star clusters. The trend may continue to even higher densities. Figure 1 summarizes the observations. 


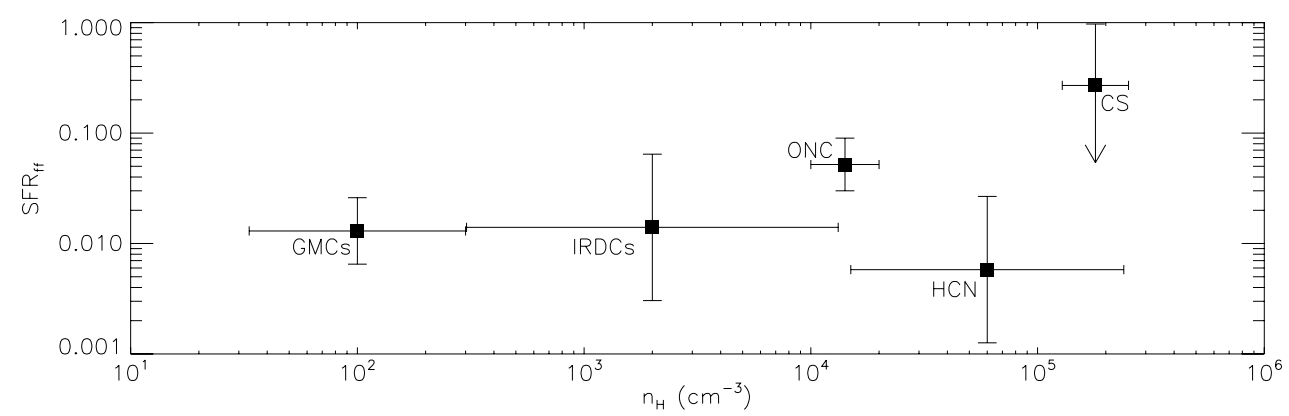

Figure 1. Fraction of mass converted into stars per free-fall time $\left(\mathrm{SFR}_{\mathrm{ff}}\right)$ versus characteristic density for various objects. We show GMCs, infrared dark clouds (IRDCs), HCN-emitting gas clumps, the Orion Nebula Cluster, and CS-emitting gas clumps. Note that the CS point is only an upper limit. The Figure is adapted from Krumholz \& Tan (2006).

The apparent universality of this factor of 100 discrepancy over such an immense range of densities and galactic environments suggests it must be set by fundamental physics. In these proceedings I present a model that attempts to explain the observations as a result of the properties of supersonic turbulence, and in which that turbulence is itself a sideeffect of the star formation process. This theory has two parts: a physical mechanism by which turbulence determines the factor of 100 , described in $\S 2$, and a physical mechanism for generating the turbulence, described in $\S 3$.

\section{Turbulent regulation of the star formation rate}

Krumholz \& McKee (2005) propose a simple model for how turbulence regulates the star formation rate, based on the premise that star formation occurs in any sub-region of a molecular cloud in which the gravitational potential energy exceeds the kinetic energy in turbulent motions. This is sufficient to determine the star formation rate in a supersonically turbulent isothermal medium, because such media are governed by two universal properties. First, they obey a linewidth-size relation, meaning that the velocity dispersion over a region of size $\ell$ varies as roughly $\ell^{1 / 2}$ (Larson 1981). Second, they show a lognormal distribution of densities (Padoan \& Nordlund 2002).

These determine the star formation rate as follows: the linewidth-size relation sets the kinetic energy per unit mass in any given sub-region of a cloud, normalized to the cloud's total kinetic energy. From this, one can show that the potential energy will be larger than the kinetic energy in regions where the density exceeds a certain critical value. In turn, the density probability distribution determines what fraction of the mass is at densities larger than this critical value. Bound regions collapse on a free-fall time scale. This determines the dimensionless star formation rate $\mathrm{SFR}_{\mathrm{ff}}$, defined as the fraction of its mass that a gas cloud turns into stars per mean-density free-fall time, in terms of two dimensionless numbers: the cloud's Mach number $\mathcal{M}$ and virial ratio $\alpha_{\text {vir }}$ (roughly its ratio of kinetic to potential energy). For $\alpha_{\text {vir }}$ and $\mathcal{M}$ in the range observed for real clouds, one may approximate the relationship by a powerlaw

$$
\mathrm{SFR}_{\mathrm{ff}} \approx 0.073 \alpha_{\mathrm{vir}}^{-0.68} \mathcal{M}^{-0.32}
$$

This is an extremely powerful result, and it allows numerous comparisons to observation. First, notice that, due to its very weak dependence on $\mathcal{M}, \mathrm{SFR}_{\mathrm{ff}}$ is a few percent in any virialized, supersonically turbulent object, regardless of its density or environment. 

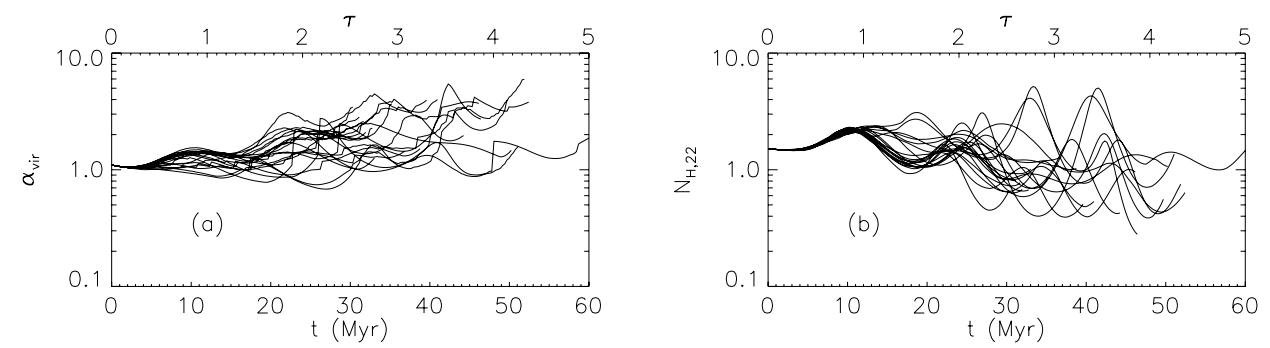

Figure 2. Virial ratio (panel a) and column density in units of $10^{22} \mathrm{~cm}^{-2}$ (panel b) versus time measured in years $(t)$ and measured in cloud crossing times $(\tau)$ for a sample of GMC evolution models. Lines end when clouds are disrupted by HII regions. The Figure is adapted from Krumholz et al. (2006a).

This is exactly what the observations summarized in Figure 1 demand; a corollary is that this model naturally explains the extra-Galactic IR-HCN correlation (Gao \& Solomon 2004; Krumholz \& Tan 2006). A second prediction of this model is that achieving the star formation efficiencies of tens of percent estimated for rich star clusters requires that star formation continue for several crossing times, a result in good agreement with the observed age spreads of young clusters (Tan et al. 2006).

A third test of the model is to use it to predict the star formation rate in the Milky Way from equation (2.1) and the observed properties of Milky Way molecular clouds. Doing so gives a predicted star formation rate of $5.3 M_{\odot} \mathrm{yr}^{-1}$ in the Milky Way, within a factor of 2 of the observed value (Krumholz \& McKee 2005). Moreover, observations of the molecular cloud populations of nearby galaxies such as M33, M64, and the LMC (see review by Blitz et al. 2005) are starting to reach precisions comparable to those available for the Milky Way. Repeating this calculation for these cloud populations and comparing to observed star formation rates provides a direct future observational test of this model.

If one adopts the additional hypotheses that molecular clouds in a galaxy should have masses of $\sim 1$ Jeans mass in the galactic disk, and should be in rough pressure balance with the rest of the ISM, then one may extend the model to predict the star formation rate in galaxies as a function of their gas surface densities, rotation rates, and molecular fractions. This prediction agrees with the data of Kennicutt (1998) as well as Kennicutt's purely empirical fit. Recent observations show that the model also agrees with the radial distribution of star formation in the Milky Way (Luna et al. 2006).

\section{Star formation regulation of the turbulence}

Having shown that turbulent regulation very naturally explains a large number of observations about star formation, we now turn to the question of the origin of the turbulence itself. This is a problem because simulations (e.g. Stone et al. 1998) show that, if it is not continually driven, supersonic turbulence will decay away over time scales much shorter than the observationally-estimated $\sim 30$ Myr lifetimes of GMCs in local group galaxies (Blitz et al. 2005). Since the clouds all have virial ratios $\sim 1$ and roughly constant column densities $\sim 10^{22} \mathrm{~cm}^{-2}$, something must be driving the turbulence and keeping the clouds in at least approximate equilibrium.

To investigate this problem, Krumholz et al. (2006a) construct simple one-dimensional semi-analytic models of GMCs, the goal of which is to investigate their global energy balance. In these models one follows the evolution of GMCs using the non-equilibrium virial and energy conservation equations for a homologously-moving, evaporating cloud, 
including source terms describing decay of turbulence at the rates measured by simulations, and a countervailing injection of energy (and removal of mass) by star formation feedback. Both energy injection and mass loss are dominated by HII regions launched by newborn star clusters (Matzner 2002), so the models focus on them.

These models show that feedback is able to explain the observed properties of GMCs extremely well. Feedback destroys giant clouds in $\sim 30 \mathrm{Myr}$, during which time they turn $5-10 \%$ of their mass into stars, and remain turbulent, virialized, and at constant column densities. Figure 2 shows some typical examples of molecular cloud evolution, which are in good agreement with observations. In contrast, clouds with masses $\sim 10^{4} M_{\odot}$, like those in the solar neighborhood, survive only $\sim 1$ crossing time. The next step in this sort of modeling is to do full ionizing radiative-transfer MHD simulations to study feedback in a more realistic context, a project already underway (Krumholz et al. $2006 \mathrm{~b})$.

\section{Summary}

Feedback-driven turbulence provides a simple, natural explanation for a host of observations about star formation. The quantitative prediction of the star formation rate one derives by computing the fraction of bound mass in a turbulent, virialized object, using no physics other than the invariant properties of supersonic isothermal turbulence, matches observations of the rate of star formation in dense gas, the rate and radial distribution of star formation in the Milky Way, the Kennicutt law, and the extragalactic IR-HCN correlation. The turbulence is in turn driven by the feedback from star formation itself. The physics of the driving process explains the observed lifetimes, column densities, and virial ratios of giant molecular clouds in local group galaxies.

\section{Acknowledgements}

I thank T. A. Gardiner, C. D. Matzner, C. F. McKee, J. M. Stone, and J. C. Tan, all of whom collaborated with me on parts of this project. I acknowledge support from NASA through Hubble Fellowship grant \#HSF-HF-01186 awarded by STScI, which is operated by AURA for NASA, under contract NAS 5-26555.

\section{References}

Blitz, L., Fukui, Y., Kawamura, A., Leroy, A., Mizuno, N. \& Rosolowsky, E. 2005, in: B. Reipurth, D. Jewitt \& K. Keil (eds.), Protostars $\&$ Planets V, in press, astro-ph/0602600

Bronfman, L., Casassus, S., May, J. \& Nyman, L.-A. 2000, A $\& A$ 358, 521

Downes, D. \& Solomon, P. M. 1998, ApJ 507, 615

Gao, Y. \& Solomon, P. M. 2004, ApJS 152, 63

Kennicutt, R. C. 1998, ApJ 498, 541

Krumholz, M. R., Matzner, C. D. \& McKee, C. F. 2006a, ApJ in press, astro-ph/0608471

Krumholz, M. R. \& McKee, C. F. 2005, ApJ 630, 250

Krumholz, M. R., Stone, J. M. \& Gardiner, T. A. 2006b, ApJ submitted, astro-ph/0606539

Krumholz, M. R. \& Tan, J. C. 2006, ApJ in press, astro-ph/0606277

Larson, R. B. 1981, MNRAS 194, 809

Luna, A., Bronfman, L., Carrasco, L. \& May, J. 2006, ApJ in press, astro-ph/0512046

Matzner, C. D. 2002, ApJ 566, 302

McKee, C. F. 1999, in NATO ASIC Proc. 540: The Origin of Stars and Planetary Systems, 29

McKee, C. F. \& Williams, J. P. 1997, ApJ 476, 144

Padoan, P. \& Nordlund, Å. 2002, ApJ 576, 870

Stone, J. M., Ostriker, E. C. \& Gammie, C. F. 1998, ApJ 508, L99

Tan, J. C., Krumholz, M. R. \& McKee, C. F. 2006, ApJ 641, L121 
Wong, T. \& Blitz, L. 2002, ApJ 569, 157

Zuckerman, B. \& Evans, N. J. 1974, ApJ 192, L149

\section{Discussion}

BONNELL: 1) As molecular clouds with and without star formation have similar turbulent properties, it would appear unlikely that outflows are the dominant process in driving turbulence. 2) The requirement for molecular clouds to last a few (2-4) crossing times is consistent with purely decaying turbulence, as the formation time of the cloud, as it must form from lower density gas, is easily several crossing times.

Krumholz: To question 1: first, while there are clouds without HII regions, there do not appear to be starless clouds. At 24 microns, all the GMCs in the LMC show embedded sources. Second, GMCs are born turbulent, and some very young ones will not have had much turbulent decay or formed many stars yet. This doesn't mean that feedback is unimportant, because all turbulence looks the same, so you can't easily tell whether it is "primordial" and feedback-driven. To question 2: recall that $30 \mathrm{Myr}$ is the time for which $>10^{5} \mathrm{M}_{\odot}$ of $\mathrm{CO}$ is visible, and $\mathrm{CO}$ only forms in clouds dense enough to be self-shielding. You could have 30 Myr correspond to 2 free-fall times if you first saw the cloud when its density was $\sim 10 \mathrm{H}$ atoms per $\mathrm{cm}^{-3}$, but at such low densities there would be no CO.

WALBORN: Your theoretical picture is in reasonable agreement with the empirical morphology of massive star-forming regions. The observation that triggered second generations are always predominantly on one side of the first generation clusters indicates that the latter form near the surface of the GMC, and not from a central collapse, perhaps caused by external events. Thus, the free-fall time of the entire GMC is irrelevant. Moreover, the difference between a super star cluster (e.g. 30 Doradus) and a seeded OB association (e.g. NII and NGC604) may arise from the bound or unbound state of the progenitor cloud subvolume, as proposed by Bally \& Zinnecker.

Krumholz: This probably gives me a bit too much credit, since my GMC model does not include explicit triggering. However, I do recover the observed result that there are generations of star formation modulated by feedback. I would disagree with a couple of your suggestions. First, I don't think the free-fall time of the entire GMC is irrelevant. There are dense sub-regions within GMCs that evolve faster, but if you want to know about the global evolution, you have to look at the bulk of the mass, and that gas evolves on the mean-density free-fall time scale. Second, I seriously doubt that the progenitors of OB associations are unbound. These clouds have virial ratios near unity, they are strongly centrally concentrated, and their morphologies are very round, observations that seem hard to reconcile with colliding, unbound gas. I'd say whether something winds up bound is likely determined by feedback. Systems that convert a large fraction of their mass into stars before disruption wind up bound, while systems that are disrupted while turning only a small percentage of their mass into stars wind up unbound.

Clark: 20 Myr is a long time in massive star formation. Would you not expect there to be supernova events in the clouds by this piont, which you have not modelled?

KRUMHOLZ: We actually did examine the effects of supernovae in our paper, and decided that they are less important than HII regions. That's because if a supernova goes off outside its parent cloud, the fraction of energy deposited in the cloud is very small. 
Supernovae that go off inside their parent clouds don't have a huge effect because the supernova shock loses most of its energy while still inside the star's HII region.

VAZquez-SEmadeni: You seem to be taking for granted that GMCs live for $\sim 30$ Myr. What is your evidence for that? If you refer to Fukui's observations of GMCs in external galaxies, about $30 \%$ of these don't have stars.

Krumholz: The evidence is indeed the observations of external galaxies by Fukui et al. (2005), Engargiola et al. (2003), and others quoted in the Blitz et al. PPV review. Regarding the $30 \%$ starless figure, you have to be careful with what is called starless. As I said in my answer to Ian, supposedly starless GMCs have plenty of embedded stars if you observe them at 24 microns. 